\title{
Finding ultracool brown dwarfs with MegaCam on CFHT: method and first results ${ }^{\star}$
}

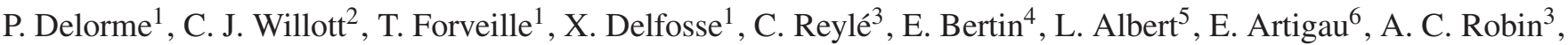 \\ F. Allard ${ }^{7}$, R. Doyon ${ }^{8}$, and G. J. Hill ${ }^{9}$
}

1 Laboratoire d'Astrophysique de Grenoble,Université J. Fourier, CNRS, UMR5571, Grenoble, France e-mail: Philippe.Delorme@obs.ujf-grenoble.fr

2 University of Ottawa, Physics Department, 150 Louis Pasteur, MacDonald Hall, Ottawa, ON K1N 6N5, Canada

3 Observatoire de Besançon, Institut Utinam, UMR CNRS 6213, BP 1615, 25010 Besançon Cedex, France

${ }^{4}$ Institut d'Astrophysique de Paris-CNRS, 98bis Boulevard Arago, 75014, Paris, France

5 Canada-France-Hawaii Telescope Corporation, 65-1238 Mamalahoa Highway, Kamuela, HI96743, USA

${ }^{6}$ Gemini Observatory Southern Operations Center c/o AURA, Casilla 603 La Serena, Chile

7 C.R.A.L. (UMR 5574 CNRS), École Normale Supérieure, 69364 Lyon Cedex 07, France

8 Département de physique and Observatoire du Mont Mégantic, Université de Montréal, CP 6128, Succursale Centre-Ville, Montréal, QC H3C 3J7, Canada

9 McDonald Observatory, University of Texas at Austin, 1 University Station C1402, Austin, TX 78712-0259, USA

Received 12 October 2007 / Accepted 2 April 2008

\section{ABSTRACT}

\begin{abstract}
Aims. We present the first results of a wide field survey for cool brown dwarfs with the MegaCam camera on the CFHT telescope, the Canada-France Brown Dwarf Survey, hereafter CFBDS. Our objectives are to find ultracool brown dwarfs and to constrain the field-brown dwarf mass function thanks to a larger sample of $\mathrm{L}$ and $\mathrm{T}$ dwarfs.

Methods. We identify candidates in CFHT/MegaCam $i^{\prime}$ and $z^{\prime}$ images using optimised psf-fitting within Source Extractor, and follow them up with pointed near-infrared imaging on several telescopes.

Results. We have so far analysed over 350 square degrees and found 770 brown dwarf candidates brighter than $z_{\mathrm{AB}}^{\prime}=22.5$. We currently have $J$-band photometry for 220 of these candidates, which confirms $37 \%$ as potential L or T dwarfs. Some are among the reddest and farthest brown dwarfs currently known, including an independent identification of the recently published ULAS J003402.77005206.7 and the discovery of a second brown dwarf later than T8, CFBDS J005910.83-011401.3. Infrared spectra of three T dwarf candidates confirm their nature, and validate the selection process.

Conclusions. The completed survey will discover $\sim 100 \mathrm{~T}$ dwarfs and $\sim 500 \mathrm{~L}$ dwarfs or $\mathrm{M}$ dwarfs later than M8, approximately doubling the number of currently known brown dwarfs. The resulting sample will have a very well-defined selection function, and will therefore produce a very clean luminosity function.
\end{abstract}

Key words. stars: low-mass, brown dwarfs - techniques: spectroscopic - techniques: photometric - methods: data analysis - surveys

\begin{abstract}
^ Based on observations obtained with MegaPrime/MegaCam, a joint project of CFHT and CEA/DAPNIA, at the Canada-France-Hawaii Telescope (CFHT) which is operated by the National Research Council (NRC) of Canada, the Institut National des Sciences de l'Univers of the Centre National de la Recherche Scientifique (CNRS) of France, and the University of Hawaii. This work is based in part on data products produced at TERAPIX and the Canadian Astronomy Data Centre as part of the Canada-France-Hawaii Telescope Legacy Survey, a collaborative project of NRC and CNRS. Based on observations made with the ESO New Technology Telescope at the La Silla Observatory under programme ID 76.C-0540(A), 77.C-0594, 77.A-0707, 78.A-0651, 78.C0629 and 79.A-0663. Based on observations obtained at the Gemini Observatory, which is operated by the Association of Universities for Research in Astronomy, Inc., under a cooperative agreement with the NSF on behalf of the Gemini partnership: the National Science Foundation (United States), the Science and Technology Facilities Council (United Kingdom), the National Research Council (Canada), CONICYT (Chile), the Australian Research Council (Australia), CNPq (Brazil) and CONICET (Argentina). Based on observations with the Kitt Peak National Observatory, National Optical Astronomy Observatory, which is operated by the Association of Universities for Research in Astronomy, Inc. (AURA) under cooperative agreement
\end{abstract}

\section{Introduction}

Brown dwarfs (BD) are of interest in many fields of stellar and planetary astrophysics, including star and planet formation theories, the physics of degenerate stellar interiors, and that of very cool stellar atmospheres. Since the discovery in 1995 of an old brown dwarf companion to a star (Nakajima et al. 1995) and a few free-floating young brown dwarfs in the Pleiades cluster (Rebolo et al. 1995), numerous isolated cold field brown dwarfs have been discovered by very wide field surveys like DENIS (DEep Near Infrared Survey Epchtein et al. 1997; Delfosse et al. 1997, 1999; Martín et al. 1999; Kendall et al. 2004), 2MASS (2 Microns All Sky Survey Skrutskie et al. 2006; Kirkpatrick et al. 1999; Burgasser et al. 2000, 2004), SDSS (Sloan Digital Sky Survey York et al. 2000; Strauss et al. 1999;

with the National Science Foundation. Based on observations made with the Nordic Optical Telescope, operated on the island of La Palma jointly by Denmark, Finland, Iceland, Norway, and Sweden, in the Spanish Observatorio del Roque de los Muchachos of the Instituto de Astrofisica de Canarias. This paper includes data taken at The McDonald Observatory of The University of Texas at Austin. 
Hawley et al. 2002; Knapp et al. 2004; Chiu et al. 2006) and UKIDSS (UKIRT Infrared Deep Sky Survey Lawrence et al. 2007; Lodieu et al. 2007). Follow up of these discoveries, and of the fewer brown dwarfs identified as companions to stars (e.g. Scholz et al. 2003), has lead to spectacular advances on (1) interaction between matter and radiation in cool dense and complex turbulent atmosphere, where molecules and dust form and dissipate; (2) stellar and planetary formation and (3) galactic structure, with the first characterization of the substellar mass function (e.g. Chabrier 2001, for an extensive review).

Much interesting work however remains to be done, and the advent of wide field cameras on large telescopes makes an unprecedented volume of the Milky Way accessible for brown dwarf searches. Here we use two large surveys with $\mathrm{MegaCam}^{1}$ on the CFHT telescope, the Canada-France-Hawaii Telescope Legacy Survey (CFHTLS ${ }^{2}$ ) and the Red-sequence Cluster Survey 2 (RCS-2; Yee et al. 2007), and complement them by additional observations to address three areas of brown dwarf physics:

(1) Detection and physics of ultracool brown dwarfs $\left(T_{\text {eff }}<\right.$ $1000 \mathrm{~K})$. As of today, observed stellar and substellar atmospheres cover a continuum of physical conditions from the hottest stars $(\sim 100000 \mathrm{~K})$ to the coolest known brown dwarf (Delorme et al. 2008, $\sim 625 \mathrm{~K}$ ). There remains a sizeable temperature gap, between these coolest brown dwarfs and the $\sim 100 \mathrm{~K}$ giant planets of the Solar System. Besides their intrinsic interest, ultracool brown dwarfs provide analogs to these planets that are not encumbered by the glare of a bright star. This will greatly help guiding the design of direct planet detection experiment, which currently have to rely on unvalidated models.

(2) Brown dwarfs in the thick disc and spheroid. The LSR (Lepine-Shara-Rich) and 2MASS surveys have recently identified the first L subdwarfs (Burgasser et al. 2003; Lépine et al. 2003), i.e. low metallicity brown dwarfs from the galactic halo population, from samples of a few hundred L dwarfs. The MegaCam survey will reach further down the halo luminosity function and may find a few T-type subdwarfs.

(3) Statistics of brown dwarfs of intermediate temperature (1000 to $1500 \mathrm{~K})$. Current estimates of the substellar Galactic mass function suggest that in the disk of the Galaxy brown dwarfs are about as numerous as stars (e.g Chabrier 2001; Cruz et al. 2007). That mass function however has significant statistical noise, which reduces its power as a constraint on star formation and galactic structure theories. At these low effective temperatures the final luminosity functions from the DENIS, 2MASS and SDSS surveys will all retain significant Poisson noise. The samples from individual searches are not easily combined since they are affected by different selection biases, so only a fraction can be used to define a robust luminosity function (e.g. Cruz et al. 2007). By almost doubling the number of known brown dwarfs, from a single survey with a well understood selection function, we will provide significantly tighter constraints on the luminosity function.

The present paper describes the overall strategy of our brown dwarf search. Section 2 discusses the observational properties which we use to identify these extremely red objects, and presents the corresponding observational material, while Sect. 3

\footnotetext{
${ }^{1}$ http://www.cfht.hawaii.edu/Instruments/Imaging/ MegaPrime/

${ }^{2}$ http://www.cfht.hawaii.edu/Science/CFHTLS/
}

describes how we generate a candidate list with minimal contamination from both observational and astrophysical artefacts. Section 4 describes the characteristics of the resulting candidates and presents spectra for a few $\mathrm{T}$ dwarfs identified early-on. We conclude with a discussion of the expected results for the completed survey.

\section{Observations}

\subsection{Observational properties of brown dwarfs}

Field brown dwarfs are extremely cool objects, with a temperature range which currently extends from $\sim 2500 \mathrm{~K}$ (early L) to $\sim 625 \mathrm{~K}$ (late T) (Delorme et al. 2008; Golimowski et al. 2004; Warren et al. 2007). Even cooler, yet to found, brown dwarfs should close the temperature gap between late type $\mathrm{T}$ dwarfs and solar system giant planets $(\sim 100 \mathrm{~K})$. Brown dwarfs spectra very much differ from a black body, and have considerable structure from deep absorption lines and bands. Their spectral energy distribution (in $v F_{v}$ units) peaks in the near infrared (hereafter NIR), particularly in the $J$ photometric band, and they are most easily detected in that wavelength range. Their pure NIR JHK colours however do not very effectively distinguish them from other classes at modest $\mathrm{S} / \mathrm{N}$ ratio. Brown dwarfs are more easily recognised by including at least one photometric band below $1 \mu \mathrm{m}$, since their steep spectral slope at those wavelength produce very distinctively red $i^{\prime}-z^{\prime}$ and $z^{\prime}-J$ colours. As one recent example, the T8.5 ULAS 0034 has $\left(i^{\prime}-z\right)_{\mathrm{AB}}^{\prime}>3.0(5 \sigma$, Delorme et al. 2008), and at any S/N ratio where it is safely detected it cannot be confused with anything, except a slightly earlier T dwarf or a $z=6$ quasar. The UKIDSS discovery observation however was less than $3 \sigma$ away from the $K$ dwarf locus. Since $K$ dwarfs outnumber T dwarfs by orders of magnitude in any flux limited sample, that distance would have been woefully insufficient for a secure identification. The non-detection of ULAS 0034 at $i^{\prime}$ and $z^{\prime}$ in the deep SDSS stripe 82 played a major role in its identification by Warren et al. 2007), and other near-IR searches for brown dwarfs similarly use some $<1 \mu \mathrm{m}$ imaging to weed out their contamination.

We take advantage of the wide field of view of the MegaCam camera (Boulade et al. 2003) on the CFHT telescope, and of the trove of observational material obtained with that instrument, to select brown dwarfs on their $i^{\prime}-z^{\prime}$ colour. The $i^{\prime}-z^{\prime}$ colour has excellent dynamics for brown dwarfs, varying from 1.7 to 4.0 between mid-L and late-T (Fig. 3). It therefore provides (at least at high $\mathrm{S} / \mathrm{N}$ ratio) a good spectral type estimator. At the high galactic latitude of our survey, the $i^{\prime}-z^{\prime}$ colour distinguishes brown dwarfs from almost every astronomical source type, but it leaves one contaminant, quasars at $z \geq 5$.8. Those are of considerable interest in their own right, but need to be distinguished from the brown dwarfs. As first shown by Fan et al. (2001), the $i^{\prime}-z^{\prime}$ vs. $z^{\prime}-J$ colour/colour diagram very effectively separates the two populations (Fig. 1 and Willott et al. 2005). The very red $i^{\prime}-z^{\prime}$ of high redshift quasars is caused by deep Lyman $\alpha$ absorption on a relatively flat intrinsic spectrum), and they therefore have a more neutral $z^{\prime}-J$. The spectral distribution of brown dwarfs, in contrast, keeps rising steeply into the $J$ band. We therefore complement our MegaCam $i^{\prime}$ and $z^{\prime}$ photometry by pointed $J$-band imaging of the candidates selected on $i^{\prime}-z^{\prime}$. Besides pinpointing the (few) quasars, the $J$-band photometry very effectively rejects any remaining observational artefact, as well as the (more numerous) moderately red stars scattered into the brown dwarf/quasar box by large noise excursions. 


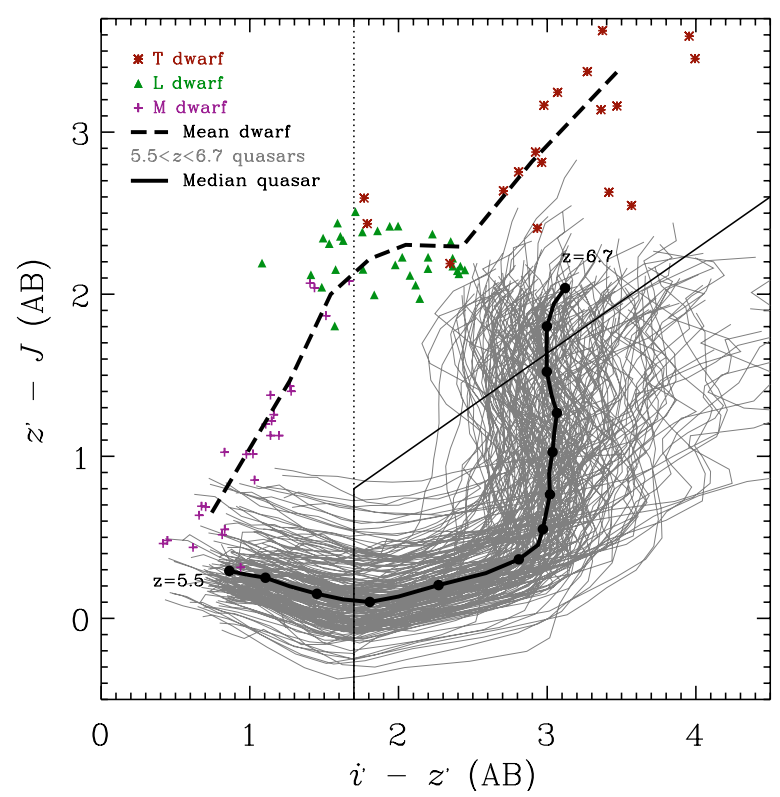

Fig. 1. $z^{\prime}-J$ and $i^{\prime}-z^{\prime}$ colours of brown dwarfs and quasars. These synthetic colours were computed for the MegaCam $i^{\prime}$ and $z^{\prime}$ photometric system and the NTT SOFI $J_{\text {short }}$ system. The bold lines represents the average colours of dwarfs (dashed) and quasars (solid). Symbols marks the individual colours of known brown dwarfs. The thin grey lines mark the colour evolution of individual synthetic quasars (described in detail in Willott et al. (2005)) as their redshift ranges from 5.5 to 6.7. The quasar redshift increases for redder colours. The vertical line at $i^{\prime}-z^{\prime}=$ 1.7 marks our $i^{\prime}-z^{\prime}$ selection criterion. The quasar selection box is also marked (to the lower-right of the solid line).

\subsection{Synthetic colours}

Each square-degree MegaCam image contains a few hundred thousand objects, of which at most a few are brown dwarfs. We thus need to strike a careful balance between sample completeness and contamination. To tune this compromise we need a precise knowledge of the colours of brown dwarfs and quasars for the exact instruments and filters used in our survey. As discussed in Willott et al. (2005), these colours are known for some photometric systems, in particular SDSS and 2MASS, but the filters and quantum efficiency curves of MegaCam are notably different (Fig. 2). This is particularly significant for brown dwarfs and quasars. Due to their highly structured spectra a modest change to a response curve can produce significantly different colours when it includes or excludes a major absorption band or emission line. We update the synthetic colours of Willott et al. (2005), using additional brown dwarfs spectra which have become available since 2005, and adding the many near-IR instruments and filters which we use for the $J$-band imaging. We use publically available spectra (from S. Leggett's website ${ }^{3}$, Martín et al. 1999; Kirkpatrick et al. 2000; Geballe et al. 2001; Leggett et al. 2002; Burgasser et al. 2003; Knapp et al. 2004; Golimowski et al. 2004; Chiu et al. 2006) of over 60 brown dwarfs with spectral types L1 to T8 (on the Burgasser et al. 2006, spectral type scale) and the synthetic quasar spectra of Willott et al. (2005). We compute their synthetic MegaCam $i^{\prime}$ and $z^{\prime}$ photometry in the AB system (Fukugita et al. 1996) using detector quantum efficiency and transmission curves for the atmosphere, telescope, camera optics, and filters (cf. Fig. 2), obtained from the CFHT web page. Figure 3 displays the resulting colours as a function of the spectral type.

\footnotetext{
$\overline{3}$ http://www.jach.hawaii.edu/ skl/LTdata.html
}

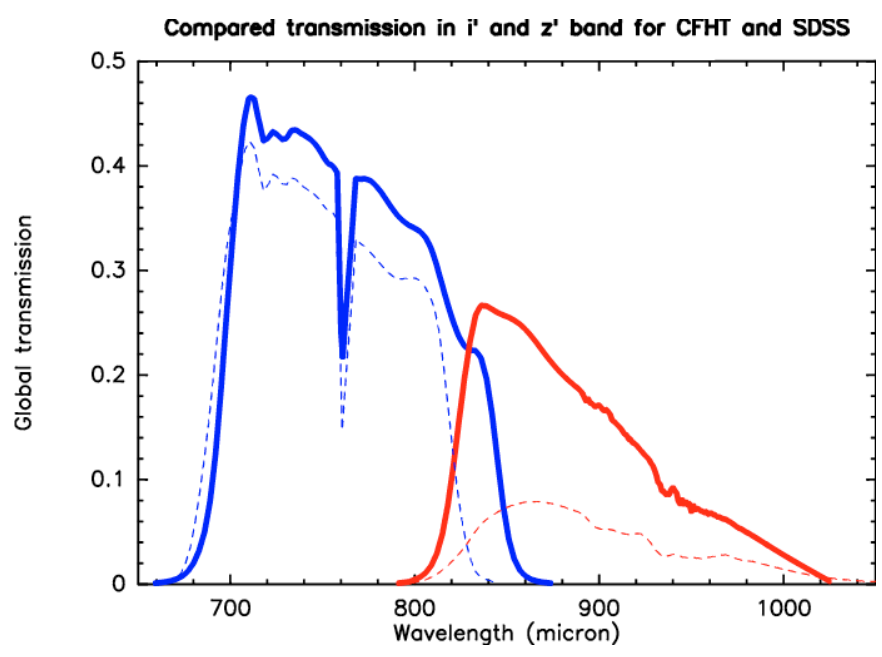

Fig. 2. Compared spectral response functions of the CFHT (thick lines) and SDSS (thin dashed lines) instruments for their $i^{\prime}$ (dark blue) and $z^{\prime}$ (red) filters. These factor in the average atmospheric transmission of the two observatory sites, the telescope reflectivities, the transmissions of the camera optics and filters, and the quantum efficiencies of the CCDs. Contrary to the SDSS bandpasses, the CFHT $i^{\prime}$ and $z^{\prime}$ filters overlap significantly, leading to less contrasted colours.

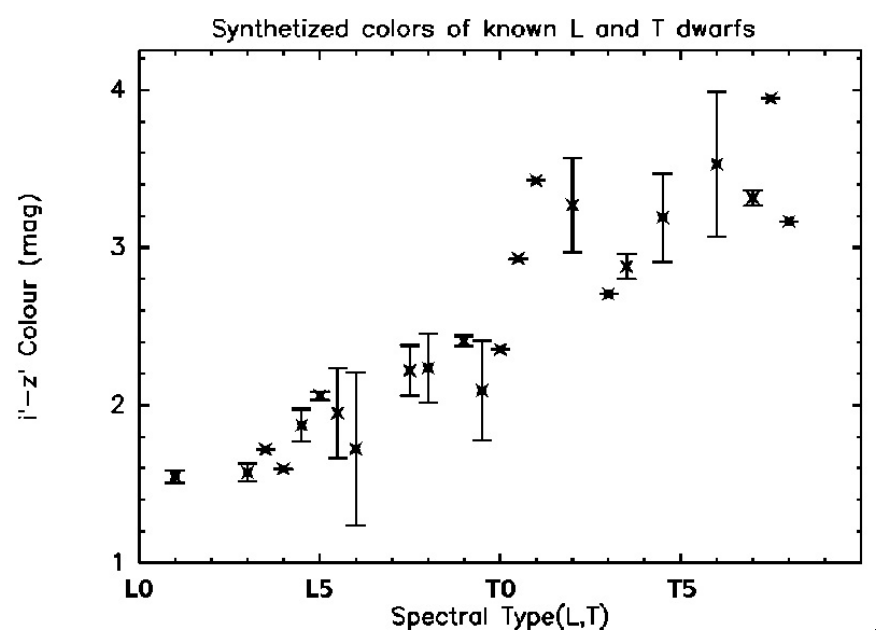

Fig. 3. $i^{\prime}-z^{\prime}$ synthetic colour versus spectral type for the MegaCam photometric system. The colour for a spectral type is the average over the brown dwarf spectra from Chiu et al. (2006), Golimowski et al. (2004) and Knapp et al. (2004) with that spectral type, and the error bars represent the dispersion (set to 0 when only one template per spectral bin is available).

We similarly synthesize $J$-band photometry for each of the instruments and $J$ filters used in the $J$-band follow up. These instruments have significantly different response curves, which must be taken into account to obtain homogeneous selection criteria. We found, in particular, that brown dwarfs colours which include $J$ photometry obtained at the NTT with SOFI and its (default) wide $J$ filter are not as red as we initially expected: contrary to most $J$ filters, its wide bandpass includes water vapor bands which are strongly absorbed in L and (particularly) T dwarfs. As a result, the $i^{\prime}-J$ and $z^{\prime}-J$ colours which use this filter are bluer by $\sim 0.15$ mag for early $\mathrm{L}$ and $\sim 0.5$ mag for late T. After we realised this we switched our SOFI observations to the alternate $J_{\text {short }}$ filter, which better separates $\mathrm{T}$ dwarfs from quasars. We use the synthetic colours to shift our selection boxes according to the actual filter. 


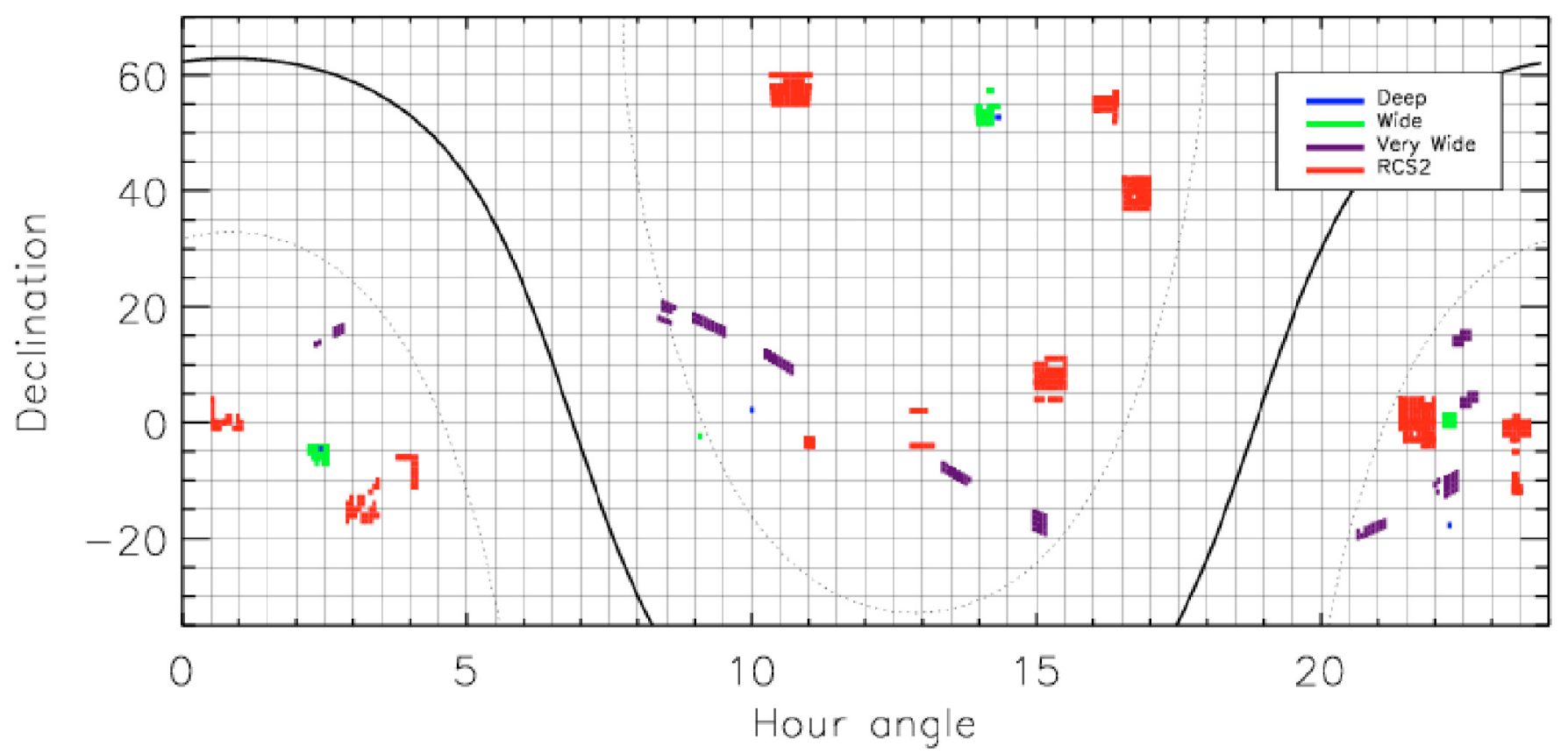

Fig. 4. Sky chart of sky area covered by CFBDS so far. Black curve marks the galactic plane while dotted curves mark $+30^{\circ}$ and $-30^{\circ}$ galactic latitude.

\subsection{Optical data}

Our survey for brown dwarfs, the Canada-France Brown Dwarf Survey (CFBDS), builds upon two major MegaCam surveys, the Canada-France-Hawaii Telescope Legacy Survey (hereafter CFHTLS) and the Red-sequence Cluster Survey 2 (hereafter RCS-2, Yee et al. 2007) and, where necessary, complements their filter coverage with the additional MegaCam observations needed to obtain pairs of $i^{\prime}$ and $z^{\prime}$ images. Figure 4 summarizes the currrent sky coverage of our optical survey data.

The CFHTLS survey has three components, named Deep, Wide, and Very Wide, (described in detail on the CFHTLS web page, http://www.cfht.hawaii.edu/ Science/CFHLS/), and we use all three.

CFHTLS Deep. This deepest component of the CFHTLS covers four high galactic latitude and low extinction 1 square degree fields in the $u^{*} g^{\prime} r^{\prime} i^{\prime} z^{\prime}$ filters, and it is primarily motivated by the SNLS type Ia supernovae search (Pain \& SNLS Collaboration 2003). The expected total exposures times per field of the completed survey will be $66 \mathrm{~h}$ at $z^{\prime}$ and $132 \mathrm{~h}$ at $i^{\prime}$ with $10 \sigma$ depths of $z_{\mathrm{AB}}^{\prime} \sim 26.25$ and $i_{\mathrm{AB}}^{\prime} \sim 27.65$. This sensitivity is sufficient to identify mid-L dwarfs out to 1300 parsecs. At such distances, and at high galactic latitude, the thick disk becomes dominant and we therefore have good hopes to probe its brown dwarf population. The present analysis uses the T0003 release (T0004 was very recently released, but we have yet to analyse and follow-up the corresponding detections) which has $10 \sigma$ depths of $z_{\mathrm{AB}}^{\prime} \sim 24.7$ and $i_{\mathrm{AB}}^{\prime} \sim 26.25$. Thanks to their large number of contributing exposures and their extended time base, the T0003 images are extremely clean, with essentially no contamination by cosmic rays or bad pixels, or by variable or moving objects such as supernovae and asteroids.

CFHTLS Wide. This component of the CFHTLS will cover $186 \mathrm{deg}^{2}$ divided between four high galactic latitude low extinction fields, in $u^{*} g^{\prime} r^{\prime} i^{\prime} z^{\prime}$, and it is primarily motivated by cosmological weak lensing. We have analysed the $20 \mathrm{deg}^{2}$ that have both $i^{\prime}$ and $z^{\prime}$ coverage in the T0003 CFHTLS release ( $i^{\prime}$ coverage is considerably more extensive, due to priorities set by the main drivers of the Wide survey). The average $10 \sigma$ depths are $z_{\mathrm{AB}}^{\prime} \sim 23.8$ (for total exposure times of $7200 \mathrm{~s}$ ) and $i_{\mathrm{AB}}^{\prime} \sim 24.75$ (for total exposure times of $4300 \mathrm{~s}$ ), with small field to field variations due to seeing and sky background fluctuations. Of the three components the Wide probes the largest volume. The Wide images have enough coadded subexposures (9 for $z^{\prime}$ and 7 for $i^{\prime}$ ) to reject all cosmic rays and bad pixels, and the overall exposure times are sufficiently long to eliminate all but the slowest moving objects. The $i^{\prime}$ and $z^{\prime}$ images on the other hand are usually not contemporaneous, and variable sources (in practice mostly supernovae) which are serendipitously bright in the $z^{\prime}$ can erroneously pass our $i^{\prime}-z^{\prime}$ colour filter. Those need to be eliminated at a later stage.

CFHTLS Very Wide (VW). This shallowest component of the CFHTLS is motivated by transneptunian objects and was initially set to cover $1000 \mathrm{deg}^{2}$ in the ecliptic plane with $g^{\prime} r^{\prime} i^{\prime} \mathrm{im}$ ages. It was later downsized to $\sim 250 \mathrm{deg}^{2}$ when it was realised that the three components could not all be completed within the allocated time. We use the $\sim 150 \mathrm{deg}^{2}$ from the Very Wide with absolute value of the galactic latitude above 30 degrees to ensure low absorption (see Table 1). The average $10 \sigma$ depth of the $540 \mathrm{~s}$ $i^{\prime} \mathrm{VW}$ exposures is $i_{\mathrm{AB}}^{\prime} \sim 23.95$, and we complement them by $420 \mathrm{~s} z^{\prime}$ exposures, with typical $10 \sigma$ depths of $z_{\mathrm{AB}}^{\prime} \sim 22.8$. Both sets of images are coadditions of 3 subexposures separated by at least one night. We therefore have enough information to reject the vast majority of bad pixels, cosmic ray hits, and moving solar system objects. The time span of the $3 z^{\prime}$ exposures on the other hand is usually too short to reliably recognize supernovae, which vary on time scales of a few weeks. We therefore again need to reject these contaminants at a later stage.

Red-sequence Cluster Survey 2 (RCS-2). The RCS-2, designed to identify distant galaxy clusters through their galaxies on the red sequence, (Yee et al. 2007) is an ongoing $g^{\prime} r^{\prime} z^{\prime}$ survey of $800 \mathrm{deg}^{2}$ at high galactic latitude to lower the absorption (see Table 1 . We have to date used $\sim 600 \mathrm{deg}^{2}$ kindly made available to us by the RCS-2 consortium, and we complement their $360 \mathrm{~s}$ $z^{\prime}$ band images by $500 \mathrm{~s}$ or $680 \mathrm{~s} i^{\prime}$ exposures, depending on the seeing. The resulting $10 \sigma$ depths of $\left(z_{\mathrm{AB}}^{\prime} \sim 22.5\right.$ and $i_{\mathrm{AB}}^{\prime} \sim 24.0$ 
Table 1. Characteristics of the optical surveys used by CFBDS.

\begin{tabular}{|c|c|c|c|c|c|c|c|c|}
\hline Survey Name & $\begin{array}{l}z^{\prime} \text { detection } \\
\text { limit }\end{array}$ & $\begin{array}{l}i^{\prime} \text { detection } \\
\text { limit }\end{array}$ & $\begin{array}{l}\text { Mid-L de- } \\
\text { tection } \\
\text { range(pcs) }\end{array}$ & $\begin{array}{l}\text { Early-T } \\
\text { detection } \\
\text { range(pcs) }\end{array}$ & $\begin{array}{l}\text { Late-T } \\
\text { detection } \\
\text { range }(\mathrm{pcs})\end{array}$ & $\begin{array}{l}\text { current cover- } \\
\text { age (sq deg) }\end{array}$ & $\begin{array}{l}\text { final cov- } \\
\text { erage(sq } \\
\text { deg) }\end{array}$ & $\begin{array}{l}\text { Galactic } i^{\prime}-z^{\prime} \\
\text { Reddening }\end{array}$ \\
\hline RCS-2 & 22.5 & 24.0 & 185 & 160 & 45 & 200 & 600 & $0.011 \pm 0.009$ \\
\hline $\begin{array}{l}\text { CFHTLS } \\
\text { Very Wide }\end{array}$ & 22.8 & 23.95 & 215 & 180 & 50 & 150 & 150 & $0.020 \pm 0.009$ \\
\hline $\begin{array}{l}\text { CFHTLS } \\
\text { Wide }\end{array}$ & 23.8 & 24.75 & 340 & 290 & 80 & 20 & 186 & $<0.02$ \\
\hline $\begin{array}{l}\text { CFHTLS } \\
\text { Deep }\end{array}$ & 24.5 & 26.25 & 470 & 400 & 110 & $\begin{array}{l}3.8 \text { to } z^{\prime}= \\
24.5\end{array}$ & $\begin{array}{l}3.8 \text { to } z^{\prime}= \\
26.3\end{array}$ & $<0.02$ \\
\hline
\end{tabular}

The detection limits correspond to $10 \sigma$, as needed for $10 \%$ precision photometry.

are similar to those of the CFHTLS-VW. Both the RCS-2 images and our complementary $i^{\prime}$ data are single exposures, which maximize the depth achieved for a given observing time. We use the RCS-2 $g^{\prime}$ and $r^{\prime}$ images, which are contemporaneous with the $z^{\prime}$ ones, to identify and reject both supernovae and moving solar system objects.

All images are pre-processed by the CFHT staff using the ELIXIR package (Magnier \& Cuillandre 2004). The CFHTLS Deep and Wide images are aligned and coadded by the Terapix data center (Bertin et al. 2002). For the CFHTLS Very Wide and RCS-2 datasets, we carry out our own processing to check and refine the astrometry and (for fields which overlap the SDSS) photometry. For the CFHTLS Very Wide, each pointing has 3 subexposures per filter which are combined whilst rejecting bad pixels and cosmic ray impacts. The CFHTLS Very Wide and RCS-2 images in different filters are aligned (with distortion correction) and trimmed to their common area.

To date most of our volume coverage originates in the CFHTLS Very Wide and RCS-2, due in part to the late start of the $z^{\prime}$ part of CFHTLS-Wide. We have currently analysed $350 \mathrm{deg}^{2}$ of the $800 \mathrm{deg}^{2}$ expected for these two surveys. Their relative shallowness has the advantage of producing targets for which spectroscopy can be obtained relatively easily on $8 \mathrm{~m}$-class telescopes.

Table 1 summarizes the properties of the four surveys, listing the limiting magnitude, the maximum distances at which mid$\mathrm{L}$ and late-T dwarfs can be detected to these magnitudes, and the current and final areas covered by the survey. Our full survey probes several times the SDSS volume for T dwarfs and we expect to detect $\sim 100$ new $\mathrm{T}$ dwarfs (compared with the $\sim 150$ currently known)

\subsection{Near infrared imaging}

As explained above, we use $J$-band photometry to distinguish between brown dwarfs and $z>5.8$ quasars. For brown dwarfs (and very low mass stars) the $z^{\prime}-J$ colour also provide a good spectral type diagnostic, for which we obtain better $\mathrm{S} / \mathrm{N}$ ratio than $i^{\prime}-z^{\prime}$. That is in particular very helpful in eliminating midM dwarfs scattered into our $i^{\prime}-z^{\prime}$ selection box by several $\sigma$ noise excursions. Given the relative numbers of mid-M stars and brown dwarfs in a magnitude-limited sample, these noise excursions are sufficiently frequent to very significantly contaminate our $i^{\prime}-z^{\prime}$ selection, but the better $\mathrm{S} / \mathrm{N}$ ratio of the $z^{\prime}-J$ colour (and the low likelihood of large noise excursions at both $i^{\prime}-z^{\prime}$ and $z^{\prime}-J$ ) makes them obvious once we obtain $J$-band images.

The $J$-band follow up has been carried out at several observatories: La Silla (NTT, 3.6 m), McDonald (2.7 m), Kitt Peak (2.1 m), La Palma (NOT, $2.5 \mathrm{~m}$ ), see Table 2. We adjust our integration times to achieve either a clear detection or a limiting magnitude that excludes any dwarf and demonstrates that the candidate is a high redshift quasar (usually around $J_{\mathrm{AB}}=22$ ). For the few candidates which are not detected at $i^{\prime}$ and where we cannot exclude that the object was a supernovae in the $z^{\prime}$ image, we integrate deeper to detect the quasar at $J$. Any supernova has long faded, and cannot be detected at any reasonable depth.

At the NTT, which accounts for most of our $J$-band follow up, we usually need exposures times of 5 to $10 \mathrm{~min}$, obtained as $\sim 40 \mathrm{~s}$ individual exposures which we jitter to measure and substract the sky background. The typical integration time on 2 meter-class telescope was $30 \mathrm{~min}$.

\section{Candidate selection}

Since brown dwarfs and high-redshift quasars are extremely red, and since our $i^{\prime}$ images are only moderately deeper than the $z^{\prime}$ ones, some of the most interesting targets are only detected at $z^{\prime}$ (i.e. are $i^{\prime}$-dropouts). Any unrecognised artefact (cosmic ray impacts, unflagged hot pixel, optical ghost, etc.) in a $z^{\prime}$ image therefore translates into an invalid brown dwarf/quasar candidate, since it associates a $z^{\prime}$ detection with (usually) an $i^{\prime}$ upper limit. Since brown dwarfs are very rare $\left(\sim 1\right.$ per $1 \mathrm{deg}^{2} \mathrm{MegaCam}$ image, which contains from 50000 to over 300000 astronomical sources), false detection rates of even 1 per $10^{4}$ real sources would swamp true brown dwarfs in our candidate lists. We therefore need to very effectively reject artefacts. Most of these are visually obvious, and we do examine every candidate before scheduling any follow-up observations, but the many hundred 340 Megapixel images which we analyse contain too many artefacts for this to be a practical first line of defense.

Fortunately, high-redshift quasars (at the resolution of the MegaCam images) and brown dwarfs are point-like. We therefore only need to distinguish point-like sources from both artefacts and extended objects, and don't have to tackle the much more difficult task of separating general astronomical sources from artefacts. Point Spread Function (hereafter PSF) fitting provides an excellent stellarity diagnostic, as well as optimal photometry and astrometry for point sources. It therefore forms the basis of our selection procedure.

\subsection{Image analysis}

We use the well known SExtractor (Bertin \& Arnouts 1996) photometry package, to which two of us (Bertin \& Delorme, in prep.) recently added a PSF-fitting module. In keeping with the general SExtractor philosophy, this module implements a dual-image mode, where source positions in a "detection image" precisely determine where photometry will be extracted in 
Table 2. Technical characteristics of the telescopes used for the $J$-band follow up.

\begin{tabular}{llllll}
\hline \hline Telescope Name & Diameter(m) & Instrument & $\begin{array}{l}\text { Exp time for Pixel scale } \\
5 \sigma \text { detection of } \\
J(\mathrm{AB})=21 \text { object }\end{array}$ & Field of view \\
\hline NTT & 3.6 & SOFI & $45 \mathrm{~s}$ & $0.288^{\prime \prime}$ & $4.9^{\prime} \times 4.9^{\prime}$ \\
\hline Kitt Peak 2.1 m & 2.1 & SQIID & $\sim 320 \mathrm{~s}$ & $0.69^{\prime \prime}$ & $5.1^{\prime} \times 5.3^{\prime}$ \\
\hline $\begin{array}{l}\text { Harlan-Smith } \\
\text { Telescope }\end{array}$ & 2.7 & ROKCAM & $\sim 3000 \mathrm{~s}$ & $0.35^{\prime \prime}$ & $1.5^{\prime} \times 1.5^{\prime}$ \\
\hline NOT & 2.5 & NOTCam & $\sim 100 \mathrm{~s}$ & $0.24^{\prime \prime}$ & $4.0^{\prime} \times 4.0^{\prime}$ \\
\hline
\end{tabular}

a "photometry image". This dual image mode is particularly well matched to the extreme colours of our targets: given the relative depths of the $i^{\prime}$ and $z^{\prime}$ images, any object of interest is very robustly detected at $z^{\prime}$ but faintly, if at all, at $i^{\prime}$. We therefore use the $z^{\prime}$ image as the detection image for both $i^{\prime}$ and $z^{\prime}$, naturally producing matched catalogues of $i^{\prime}$ and $z^{\prime}$ photometry for every object that is well detected at $z^{\prime}$, independently of its $i^{\prime}$ significance. This eliminates the delicate task of handling unmatched sources in independent catalogues: those might be weakly detected in the $i^{\prime}$ image, though with too low a significance for inclusion in any modestly reliable single image $i^{\prime}$ catalogue, and they therefore cannot validly be handled as pure upper limits.

SExtractor implements simultaneous fitting of multiple PSFs to blended objects, providing accurate parameters for close binaries and usable measurements for point-like sources blended with galaxies. The latter is particulary important for the quasar search, since it can recover some lensed quasars which would otherwise be lost to confusion with their lensing galaxy. In addition to more accurate parameters for the affected objects, this better handling of blends produces more complete catalogues in crowded fields. Introducing multiple PSFs recovers $\sim 3 \%$ additional sources in the relatively shallow CFHTLS-VW and RCS-2 images, and $\sim 10 \%$ in the deeper CFHTLS-Deep images. PSF-fitting also improves the photometric precision by $\sim 10 \%$ over optimum aperture photometry (Bertin \& Delorme, in prep.), and it therefore allows us to use slightly deeper catalogues, for another $15 \%$ gain in sample size. Since low significance $i^{\prime}$ detections provide colours with complex error distributions, we replace them by the $5 \sigma$ detection limit on their image and compute a lower limit on $i^{\prime}-z^{\prime}$. We note that the resampling involved in the coaddition of images built from multiple exposures, and in the filter to filter alignment, generates noise correlations on scales of 1-2 pixels. Thanks to the generous sampling of our MegaCam images $\left(0.186^{\prime \prime} /\right.$ pixel and seeing mostly above $\left.0.6^{\prime \prime}\right)$ and the use of a Lanczos3 interpolation function, source profiles are negligibly affected (Bertin et al. 2002), but resampling has a measurable low-pass filtering effect on photon noise. As we decided for practical reasons to ignore noise covariances in our fitting, the net effect on photometry is that errors estimates must be multiplied by a factor $\sim 1.4$. That factor is well determined for the CFHTLS-Deep and CFHTLS-Wide images, which are built from a large number of individual exposures, but for the CFHTLS-VW images it significantly varies from field to field according to the sub-pixel relative positions of the 3 coadded exposures.

\subsection{Filtering and target selection}

We start by requiring a $>10 \sigma$ detection in the $z^{\prime}$ filter and a $i^{\prime}-z^{\prime}>1.7$. These criteria without any additional filtering typically yield over 10000 candidates per RCS-2 1 square degree field. The single exposures per filter used for the RCS-2 survey (and for our follow-up of its fields) are most affected by cosmic ray hits and bad pixels, and the stacked images used in the other components contain fewer such artefacts. Flagging of known bad-pixel positions and simple morphological rejection of cosmic ray hits lowers this number to under 1000 , but not to a point where visual examination would be practical.

We then assess the stellarity of each candidate from its SExtractor output parameters to further decrease the number of false detections. After experimenting with several parameter combination, we have converged to the quality of the fit between the image and the PSF model, as summarised by the $\chi^{2}$ of the residuals, as our main diagnostic. We found that Sextractor's default stellarity index, based on a specifically trained neural network, works well at high signal to noise ratios, but that it becomes ineffective for the faint objects which dominate our catalogues. We similarly found that tests based on comparisons of fluxes through different apertures, or on peak surface brightness versus flux, are always less distinctive than $\chi^{2}$ filtering. Since we currently prefer to visually inspect all final candidates, we very conservatively set our filtering threshold to a level where $\sim 10 \%$ of the candidates are visually acceptable. These filtering criteria typically yield under 50 candidates per square degree. A lower threshold would not very significantly decrease the inspection workload, and might conceivably eliminate a few valid candidates. We will probably revisit this tuning as we gain experience with, and confidence in, our selection process.

With our current settings, $\chi^{2}$ filtering reduces the number of artefacts by a factor of $10-15$ (Fig. 5). Visual inspection of the more than 2000 sources with $i^{\prime}-z^{\prime}>1.7$ in a 4 square degrees test region showed that $\chi^{2}$ filtering rejected none of the 14 valid point-like sources, and our resolution of the few initial discrepancies was always in favour of the $\chi^{2}$ filtering diagnostic. Further tests also verified that our current threshold is comfortably above the highest $\chi^{2}$ measured for valid candidates, and therefore very conservative.

After this pruning of the initial catalogue to just bona fide point sources, we select candidates with an $i^{\prime}-z^{\prime}$ criterion. Our synthetic photometry (Fig. 3) demonstrates that the L dwarf domain begins at $i^{\prime}-z^{\prime}>1.45$. M dwarfs however, with $i^{\prime}-z^{\prime}$ just below 1.45, massively outnumber brown dwarfs in a magnitude-limited sample. Poissonian photometric errors consequently scatter a significant number of $\mathrm{M}$ dwarfs into this $\mathrm{L}$ dwarf box. We therefore set our colour filtering to $i^{\prime}-z^{\prime}>1.7$, or nominally to later than L4. With M 8 and M 9 stars having $i^{\prime}-z^{\prime} \sim 1.35$, and assuming Gaussian noise at our $z^{\prime}$ S/N limit, this colour threshold eliminates $\sim 95 \%$ of these very late M dwarfs. Mid-M dwarfs have $i^{\prime}-z^{\prime} \sim 1.1$ and we eliminate $\sim 99.9 \%$ of them. Since brown dwarfs are intrinsically much rarer, our candidate list nonetheless has some significant contamination by late-M dwarfs, but at a level which no longer overwhelms our follow-up capacity. 


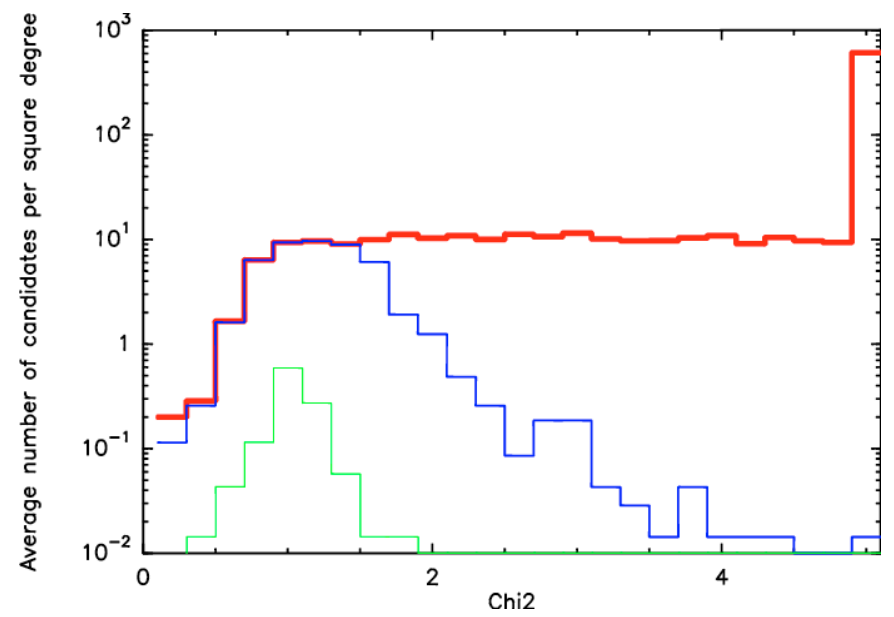

Fig. 5. Histogram of the $\chi^{2}$ of the psf fitting residuals. The thick red histogram shows all $i^{\prime}-z^{\prime}>1.7$ candidates after basic filtering, the medium-thick blue histogram those that additionally survived $\chi^{2}$ filtering, and the thin green one the final candidates retained after visual inspection and supernovae rejection. These histograms show candidates from a $70 \mathrm{deg}^{2}$ test area.

Our final filtering step is to eliminate as many as we can of the candidates which actually are supernovae that were bright at the time of the $z^{\prime}$ image. All RCS-2 fields have contemporaneous $g^{\prime}, r^{\prime}$ and $z^{\prime}$ images, and for supernovae the $g^{\prime}$ and $r^{\prime}$ images are very significantly deeper than the $z^{\prime}$ one. We therefore very reliably reject their supernovae by inspecting these $g^{\prime}$ and $r^{\prime}$ images. The CFHTLS-Deep images are stacks of exposures obtained over several years, and any supernova is eliminated by the sigma-clipping applied during stacking. The exposures which contribute to our CFHTLS-Wide and CFHTLS-VW $z^{\prime}$ images, on the other hand, were usually obtained over shorter time spans than the $\sim 6$ weeks (Pain \& SNLS Collaboration 2003) timescale of supernovae photometric evolution. We therefore mostly cannot recognize their supernovae based on their photometric variation between the individual exposures, and the exposures in other filters are usually not sufficiently contemporaneous to reject them based on a blue instaneous colour. We must therefore handle some supernovae contamination at a later stage.

\section{Results}

\subsection{Candidates}

We have so far analysed images for $357 \mathrm{deg}^{2}$, in which we have identified 770 brown dwarf and quasar candidates. We have so far extracted $J$-band photometry for 215 of those, prioritising the reddest candidates (Fig. 6).

This first set of $i^{\prime} z^{\prime} J$ photometry allows a good assessment of the actual nature of these 215 candidates. As summarised in Table 3, they include 23 likely $\mathrm{T}$ dwarfs, $57 \mathrm{~L}$ dwarfs and very late M dwarfs candidates (M8 and M9 dwarfs have very similar $z^{\prime}-J$ colours to L dwarfs), and at least 4 high redshift quasars (published in Willott et al. 2007). 22 targets remain undetected in deep $J$-band images and are most likely artefacts which our filtering did not catch. 109 objects have $z^{\prime}-J<1.6$ and most of those are likely $\mathrm{M}$ dwarf contaminants. Some however have $J$-band upper limits which are insufficiently deep to ascertain whether they are artefacts, quasars or Mid-M dwarfs. Those will need additional follow-up to clarify their status.

Since we prioritised the analysis of the reddest candidates, this first $i^{\prime} z^{\prime} J$ sample is strongly biased towards $\mathrm{T}$ dwarfs.

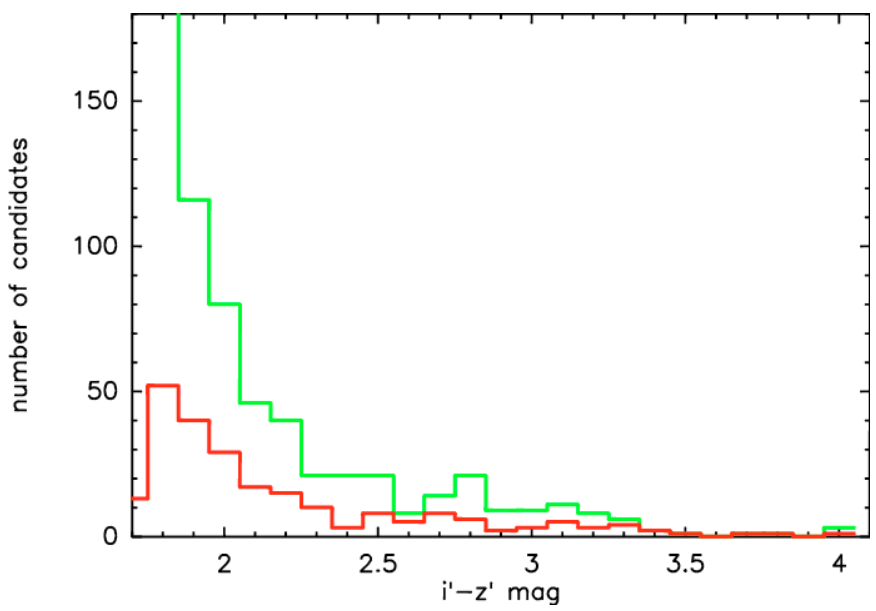

Fig. 6. Histograms of the number of $i^{\prime}-z^{\prime}$ candidates as a function of $i^{\prime}-z^{\prime}$ colour (light green), and of the number of these candidates for which $J$-band photometry is currently available (dark red).

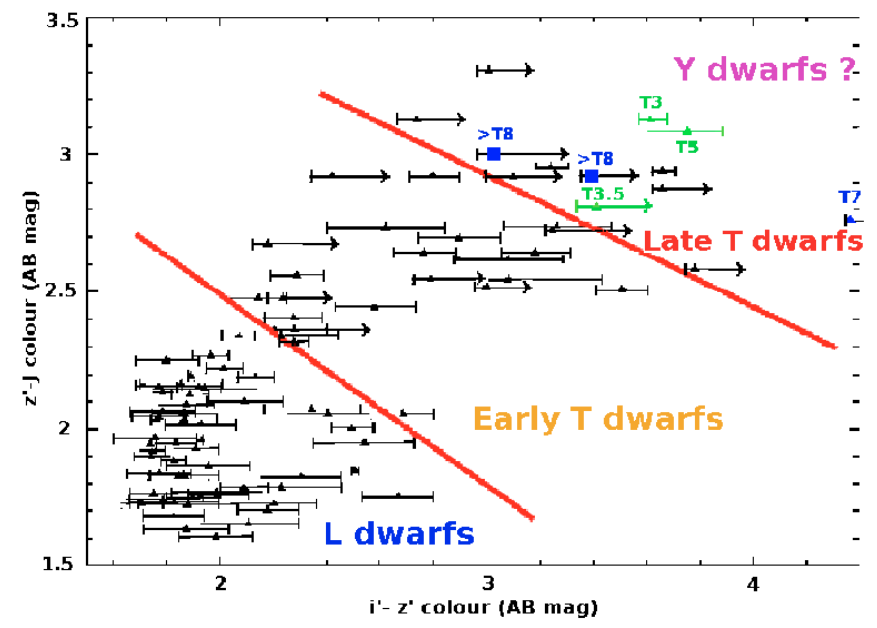

Fig. 7. $i^{\prime}-z^{\prime}$ vs. $z^{\prime}-J$ colour-colour diagram of our cool dwarf candidates (black). The error bars are $1 \sigma$ when we have $i^{\prime}$ and $z^{\prime}$ measurements. An arrow is used when $i^{\prime}-z^{\prime}$ is only a lower limit. For clarity, the $z^{\prime}-J$ errors are not shown; they are usually under 0.15 mag. The spectra of the highlighted T3, T3.5 and T5 objects are presented in this article. The big blue squares represent the coolest brown dwarfs known, ULAS J003402.77-005206.7/CFBDS J003402-005206, recently published by Warren et al. (2007) and independently identified in our survey and CFBDS J005910.83-011401.3, presented in Delorme et al. (2008). The T7 object highlighted here was published by Chiu et al. (2006) while queued for our CFBDS spectroscopy. The spectral type domains are based on our synthetic photometry.

Appproximately correcting for this bias, we estimate that $\sim 40 \%$ of our $i^{\prime}-z^{\prime}$ candidates are actual cool dwarfs, of which $\sim 15 \%$ are T dwarfs. The T dwarfs include several with extreme colours, which ongoing spectroscopic observations will characterize further. One, CFBDS J003402-005206, actually is an independent discovery of ULAS J003402.77-005206.7 which Warren et al. (2007) recently identified with the UKIDSS survey (Burningham et al. 2007) as a brown dwarf later than T8 ( 650 K). Even more recently, we obtained spectroscopic observations of an even cooler brown dwarf ( $625 \mathrm{~K}$, Delorme et al. 2008). Figure 7 shows several other candidates that are at least as promising, and those are currently queued for near-IR spectroscopy. 
Table 4. Observational properties of the three $\mathrm{T}$ dwarfs whose spectra are presented here.

\begin{tabular}{cccccccc}
\hline \hline Name & RA & Dec & $J_{\mathrm{AB}}$ & $z_{\mathrm{AB}}^{\prime}-J_{\mathrm{AB}}$ & $i_{\mathrm{AB}}^{\prime}-z_{\mathrm{AB}}^{\prime}$ & Spectral Type & Distance Range (pc) \\
\hline CFBDS100113+022622 & $10: 01: 13.1$ & $+02: 26: 22.3$ & 19.7 & 3.1 & 3.75 & $\mathrm{~T} 5 \pm 1$ & $45-110$ \\
CFBDS193430-214221 & $19: 34: 30.4$ & $-21: 42: 21.0$ & 17.9 & 2.8 & 3.6 & $\mathrm{~T} 3.5 \pm .5$ & $38-48$ \\
CFBDS095914+023655 & $09: 59: 14.8$ & $+02: 36: 55.2$ & 20.3 & 3.1 & $>3.4$ & $\mathrm{~T} 3.0 \pm .5$ & $120-130$ \\
\hline
\end{tabular}

Table 5. Spectral indices and the resulting spectral types.

\begin{tabular}{cccc}
\hline \hline Spectral Index & CFBDS100113+022622 & CFBDS193430-214221 & CFBDS095914+023655 \\
\hline $\mathrm{CH}_{4}-J$ & $0.43-\mathrm{T} 5.2$ & $0.59-\mathrm{T} 2.9$ & $0.57-\mathrm{T} 3.2$ \\
$\mathrm{H}_{2} \mathrm{O}-\mathrm{H}$ & $0.46-\mathrm{T} 3.0$ & $0.48-\mathrm{T} 2.7$ & $0.45-\mathrm{T} 3.1$ \\
$\mathrm{CH}_{4}-H$ & $0.40-\mathrm{T} 5.7$ & $0.63-\mathrm{T} 3.9$ & $0.79-\mathrm{T} 3.0$ \\
$\mathrm{CH}_{4}-K$ & $0.27-\mathrm{T} 4.7$ & $0.34-\mathrm{T} 4.1$ & $0.54-\mathrm{T} 3.0$ \\
Average Spectral Type & $\mathrm{T} 4.6 \pm 1.2$ & $\mathrm{~T} 3.4 \pm 0.7$ & $\mathrm{~T} 3.1 \pm 0.1$ \\
Weighted Spectral Type & $\mathrm{T} 5.0$ & $\mathrm{~T} 3.6$ & $\mathrm{~T} 3.1$ \\
Final Spectral Type & $\mathrm{T} 5.0 \pm 1$ & $\mathrm{~T} 3.5 \pm 0.5$ & $\mathrm{~T} 3.0 \pm 0.5$ \\
\hline
\end{tabular}

Spectral types are derived from Burgasser et al. (2006) spectral indices.

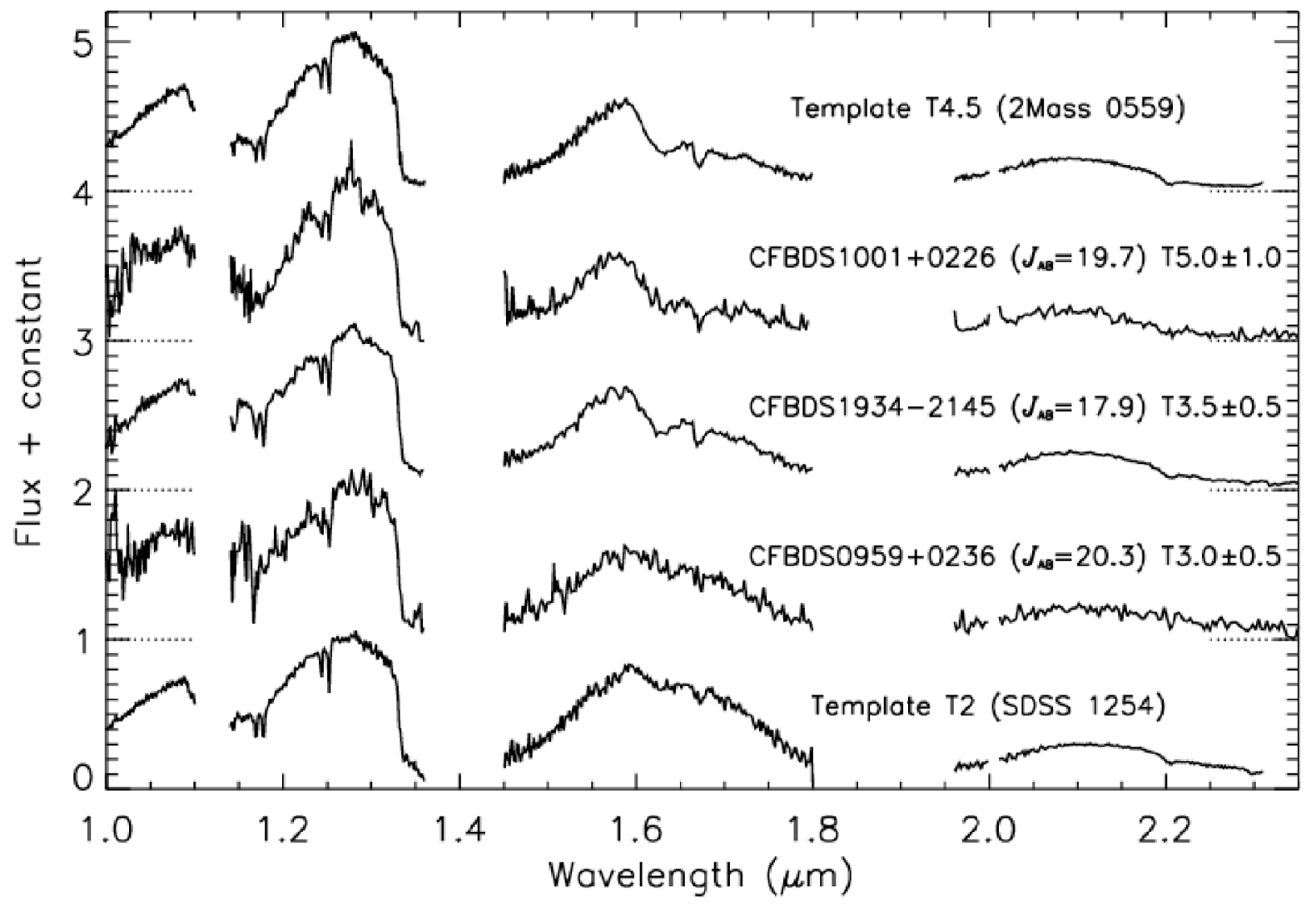

Fig. 8. GNIRS near-IR spectra of the three T Dwarfs observed on Gemini-South. The two fainter targets originate from the CFHTLS-Deep, while the brighter ones comes from the CFHTLS-VW. Templates of spectral types T2 and T4.5 from McLean et al. (2003) are shown for comparison. All spectra are normalised at 1.26 microns and displayed with integer offsets for clarity.

\subsection{Spectroscopy}

We present here near-IR spectroscopic observations of our three first $\mathrm{T}$ dwarf candidates, as an illustration of the content of our candidates catalogue. Two, which we originally published in Willott et al. (2005), originate in the CFHTLS-Deep survey and are thus fainter than most of our candidates. The last comes from the Very Wide component of the CFHTLS and is more representative. Cross dispersed spectra were obtained during semester 2006A with GNIRS (Elias et al. 2006) at Gemini South in Service mode. The slit width of 0.68 arcsec coupled with the short camera and the $31.7 \mathrm{l} / \mathrm{mm}$ grating yielded a resolving power of 900 , and the spectra have full wavelength coverage between 0.9 and 2.4 microns. A-B (not ABBA) sequences were used, with individual 5-minutes exposures for the brighter target and 10-minutes for the fainter CFHTLS-Deep targets. The total on-source integration time is $30 \mathrm{~min}$ for CF BDS 193430 $214221\left(J_{\mathrm{AB}}=17.9\right), 180$ minutes for $C F B D S 100113+022622$ 
Table 3. Preliminary classification of the candidates with $J$-band photometry.

\begin{tabular}{cccccc}
\hline \hline & T dwarfs & L dwarfs & quasars & M dwarfs & artefacts \\
\hline $\begin{array}{c}\text { Number of } \\
\text { objects }\end{array}$ & 23 & 57 & 4 & 109 & 22 \\
\hline Percentage & $10.7 \%$ & $26.5 \%$ & $1.9 \%$ & $50.7 \%$ & $10.2 \%$ \\
\hline
\end{tabular}

This classification is based on their position in the $i^{\prime}-z^{\prime}$ versus $z^{\prime}-J$ colour-colour diagram.

$\left(J_{\mathrm{AB}}=19.7\right)$ and 200 minutes for $C F B D S 095914+023655$ $\left(J_{\mathrm{AB}}=20.3\right)$. The $\mathrm{OH}$ sky lines were used for wavelength calibration, and an A-type star was observed immediately before each sequence for relative flux calibration and telluric absorption correction. The spectra were extracted and calibrated using our own IDL procedures.

The reduction proceeds as follows. The sequence of spectral images are flat-fielded using an internal flat taken immediately after the science frames. The five useful cross-dispersed orders are then extracted in five individual images that are corrected for distortion in the spectral dimension. For most objects, the trace is too faint over many wavelengths intervals to determine trace position, so its curvature is derived from the reference star spectrum. These individual order frames are then pair-subtracted, effectively removing most of the sky, dark current and hot pixels contributions. Each frame is then collapsed along the spectral dimension to determine the positive and negative traces positions. We then extract the spectra using positive and negative extraction boxes that have identical but opposite integrals; this minimizes the contribution from residual sky line that would remain from the pair subtraction. The same operation is performed on the A0 telluric calibration star. Spectra derived from individual image pairs are then median-combined into final target and calibration star spectra. A telluric absorption spectrum is then derived using the calibration star spectra. A black body spectrum with a temperature of $10000 \mathrm{~K}$ is assumed for the A0 stars and hydrogen-lines are interpolated over. The target spectrum is then divided by the derived telluric transmission spectrum. A first order wavelength calibration is obtained from an argon-lamp spectrum, and fine-tuned by registering the bright $\mathrm{OH}$-lines obtained from a sum of the pair of images of interest. Table 4 summmarizes the properties of the 3 objects.

The spectra (Fig. 8) confirm that all three candidates with spectroscopic observations are T-dwarfs. We determined their spectral types using the Burgasser et al. (2006) spectral indices. Table 5 lists thoses indices and the corresponding spectral type. We retain as our prefered determination the "weighted" spectral types, rounded to the closest half-integer. These "weighted" spectral types take into account the better sensitivity of those indices that vary most for a given subtype range. The reddest target, CFBDS100113+022622 $\left(i^{\prime}-z^{\prime}=3.75\right)$, turn out to be a T5 \pm 1 dwarf, and its 45 to 110 parsecs photometric distance makes it one of the farthest mid/late-T dwarf currently known. The large uncertainty on its distance is dominated by the spectral type uncertainty and the $>1$ dimming between T4 and T6 dwarfs, (Vrba et al. 2004), with photometric uncertainties contributing less than $5 \%$. However, the absolute magnitudes of mid-T dwarfs are themselves uncertain by as much as 1 mag (see for instance Liu et al. 2006). The faintest of the three dwarfs, CFBDS095914+023655 $\left(i^{\prime}-z^{\prime}>3.4\right)$ turns out to have an earlier spectral type, T3, but lies even farther, between 120 and 130 parsecs. The indices of the brighest one, CFBDS193430-214221 $\left(i^{\prime}-z^{\prime}=3.6\right)$, indicate a T3.5 \pm 0.5 spectral type. The spectral
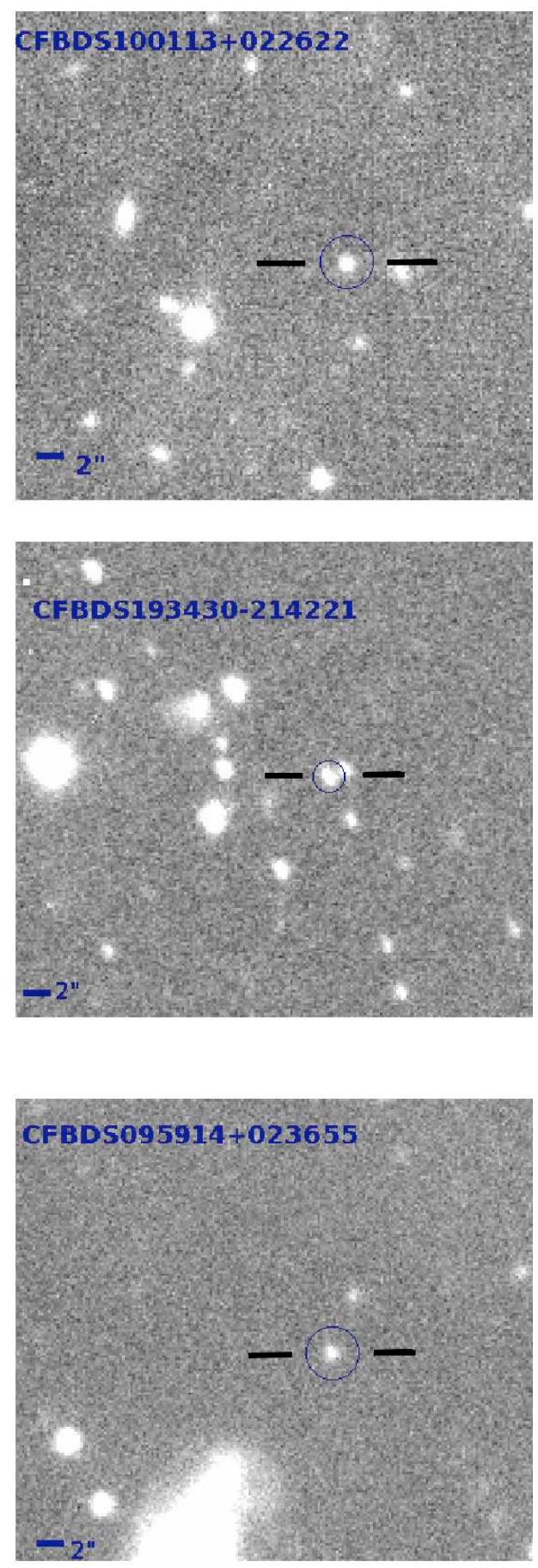

Fig. A.1. Finding charts from $z^{\prime}$ band images of the $3 \mathrm{~T}$ dwarfs whose spectra are presented in this article. The fields are $45^{\prime \prime} \times 45^{\prime \prime}$ north is up, east is left.

type uncertainties are derived from the scatter between the estimates from the various spectral indices.

\section{Conclusion}

Our survey has to date found $23 \mathrm{~T}$ dwarf candidates, $57 \mathrm{~L}$ or very-late $\mathrm{M}$ dwarf candidates, and 4 high redshift quasars, out of 215 candidates with $i^{\prime}, z^{\prime}$ and $J$ magnitudes. These were drawn from a larger sample of 770 candidates with $i^{\prime}$ and $z^{\prime}$ magnitudes, found in $357 \mathrm{deg}^{2}$ of $i^{\prime}$ and $z^{\prime}$ MegaCam images. 
Taking into account our prioritising of the reddest candidates for $J$-band observations, we expect that complete follow-up of these 770 candidates will yield $\sim 45 \mathrm{~T}$ dwarfs and $200 \mathrm{~L}$ dwarfs. Scaling to our final $\sim 800 \mathrm{deg}^{2}$ of shallow surveys, RCS-2+Very Wide, then predicts $\sim 100 \mathrm{~T}$ dwarfs and over $450 \mathrm{~L}$ or very late$\mathrm{M}$ dwarfs, approximately doubling the number of known brown dwarfs. Our analysis of the CFHTLS-Deep and CFHTLS-Wide surveys has, and will, yield additional candidates at large distances, which will constrain the galactic scale height of brown dwarfs. We plan to obtain spectra for the most exciting of these many brown dwarfs, and expect that the large discovery volume will produce even cooler objects than our recent T9/Y0 discovery, described in Delorme et al. (2008).

Acknowledgements. Thanks to the queue observers at CFHT and Gemini who obtained data for this paper (Gemini program GS-2006A-Q-16). Thanks to J.J. Kavelaars for advice on planning our MegaCam observations in the CFHTLS Very Wide and to Howard Yee and the RCS-2 team for making their proprietary data available. This research has made use of the VizieR catalogue access tool, of SIMBAD database and of Aladin, operated at CDS, Strasbourg.

\section{Appendix A: Finding charts}

See Fig. A.1.

\section{References}

Bertin, E., \& Arnouts, S. 1996, A\&AS, 117, 393

Bertin, E., Mellier, Y., Radovich, M., et al. 2002, in Astronomical Data Analysis Software and Systems XI, ed. D. A. Bohlender, D. Durand, \& T. H. Handley, ASP Conf. Ser., 281, 228

Boulade, O., Charlot, X., Abbon, P., et al. 2003, in Instrument Design and Performance for Optical/Infrared Ground-based Telescopes, ed. M. Iye, \& A. F. M. Moorwood, Proc. SPIE, 4841, 72

Burgasser, A. J., Wilson, J. C., Kirkpatrick, J. D., et al. 2000, AJ, 120, 1100

Burgasser, A. J., Kirkpatrick, J. D., Burrows, A., et al. 2003, ApJ, 592, 1186

Burgasser, A. J., Kirkpatrick, J. D., Lepine, S., et al. 2004, Am. Astron. Soc. Meet. Abstr., 205

Burgasser, A. J., Geballe, T. R., Leggett, S. K., Kirkpatrick, J. D., \& Golimowski, D. A. 2006, ApJ, 637, 1067
Burningham, B., Pinfield, D., Leggett, S. K., et al. 2007, in Am. Astron. Soc. Meet. Abstr., 210, 69.03

Chabrier, G. 2001, ApJ, 554, 1274

Chiu, K., Fan, X., Leggett, S. K., et al. 2006, AJ, 131, 2722

Cruz, K. L., Reid, I. N., Kirkpatrick, J. D., et al. 2007, AJ, 133, 439

Delfosse, X., Tinney, C. G., Forveille, T., et al. 1997, A\&A, 327, L25

Delfosse, X., Tinney, C. G., Forveille, T., et al. 1999, A\&AS, 135, 41

Delorme, P., Delfosse, X., Albert, L., et al. 2008, A\&A, in press, 802

Elias, J. H., Joyce, R. R., Liang, M., et al. 2006, in Ground-based and Airborne Instrumentation for Astronomy, ed. I. S. McLean, \& M. Iye, Proc. SPIE, 6269, 62694C

Epchtein, N., de Batz, B., Capoani, L., et al. 1997, The Messenger, 87, 27

Fan, X., Strauss, M. A., Richards, G. T., et al. 2001, AJ, 121, 31

Fukugita, M., Ichikawa, T., Gunn, J. E., et al. 1996, AJ, 111, 1748

Geballe, T. R., Saumon, D., Leggett, S. K., et al. 2001, ApJ, 556, 373

Golimowski, D. A., Leggett, S. K., Marley, M. S., et al. 2004, AJ, 127, 3516

Hawley, S. L., Covey, K. R., Knapp, G. R., et al. 2002, AJ, 123, 3409

Kendall, T. R., Delfosse, X., Martín, E. L., \& Forveille, T. 2004, A\&A, 416, L17

Kirkpatrick, J. D., Reid, I. N., Liebert, J., et al. 1999, ApJ, 519, 802

Kirkpatrick, J. D., Reid, I. N., Liebert, J., et al. 2000, AJ, 120, 447

Knapp, G. R., et al. 2004, Astron. J., 127, 3553

Lépine, S., Rich, R. M., \& Shara, M. M. 2003, ApJ, 591, L49

Lawrence, A., Warren, S. J., Almaini, O., et al. 2007, MNRAS, 379, 1599

Leggett, S. K., Hauschildt, P. H., Allard, F., Geballe, T. R., \& Baron, E. 2002, MNRAS, 332, 78

Liu, M. C., Leggett, S. K., Golimowski, D. A., et al. 2006, ApJ, 647, 1393

Lodieu, N., Pinfield, D. J., Leggett, S. K., et al. 2007, ArXiv e-prints, 705

Magnier, E. A., \& Cuillandre, J.-C. 2004, PASP, 116, 449

Martín, E. L., Delfosse, X., Basri, G., et al. 1999, AJ, 118, 2466

McLean, I. S., McGovern, M. R., Burgasser, A. J., et al. 2003, ApJ, 596, 561

Nakajima, T., Oppenheimer, B. R., Kulkarni, S. R., et al. 1995, Nature, 378, 463

Pain, R., \& SNLS Collaboration 2003, BAAS, 35, 1335

Rebolo, R., Zapatero-Osorio, M. R., \& Martin, E. L. 1995, Nature, 377, 129

Scholz, R.-D., McCaughrean, M. J., Lodieu, N., \& Kuhlbrodt, B. 2003, A\&A, 398, L29

Skrutskie, M. F., Cutri, R. M., Stiening, R., et al. 2006, AJ, 131, 1163

Strauss, M. A., Fan, X., Gunn, J. E., et al. 1999, ApJ, 522, L61

Vrba, F. J., Henden, A. A., Luginbuhl, C. B., et al. 2004, AJ, 127, 2948

Warren, S. J., Mortlock, D. J., Leggett, S. K., et al. 2007, MNRAS, 381, 1400

Willott, C. J., Delfosse, X., Forveille, T., Delorme, P., \& Gwyn, S. D. J. 2005, ApJ, 633, 630

Willott, C. J., Delorme, P., Omont, A., et al. 2007, ArXiv e-prints, 706

Yee, H. K. C., Gladders, M. D., Gilbank, D. G., et al. 2007, ArXiv Astrophysics e-prints

York, D. G., Adelman, J., Anderson, Jr., J. E., et al. 2000, AJ, 120, 1579 